\title{
Human metabolome changes after a single dose of 3,4-methylenedioxymethamphetamine (MDMA) with special focus on steroid metabolism and inflammation processes
}

\author{
Martina I. Boxlerl, Gabriel Streun ${ }^{1}$, Matthias E. Liechti ${ }^{2}$, Yasmin Schmid ${ }^{2}$, Thomas \\ Kraemer $^{l}$, Andrea E. Steuer ${ }^{*}$ \\ 1 Department of Forensic Pharmacology \& Toxicology, Zurich Institute of Forensic \\ Medicine, University of Zurich, Switzerland \\ 2 Psychopharmacology Research, Division of Clinical Pharmacology and Toxicology, \\ Department of Biomedicine, Department of Clinical Research, University Hospital Basel, \\ University of Basel, Switzerland
}

KEYWORDS 3,4-methylenedioxymethamphetamine (MDMA); ecstasy; untargeted metabolomics; pregnenolone sulfate; calcitriol; placebo-controlled; inflammation

\begin{abstract}
The intake of 3,4-methylenedioxymethamphetamine (MDMA) is known to increase several endogenous substances involved in steroid and inflammation pathways. Untargeted metabolomics screening approaches can determine biochemical changes after drug exposure and can reveal new pathways which might be involved in the pharmacology and toxicology of a drug of abuse. We analyzed plasma samples from a placebo-controlled cross-over study of a single intake of MDMA. Plasma samples from a time point before and three time points after the intake of a single dose of $125 \mathrm{mg}$ MDMA were screened for changes of endogenous metabolites. An untargeted metabolomics approach on a high resolution quadrupole time of flight mass spectrometer coupled to liquid chromatography with two different chromatographic systems (reversed phase and hydrophobic interaction liquid chromatography) was applied. Over 10 '000 features of the human metabolome were discovered. Hence, 28 metabolites were identified which showed significant changes after administration of MDMA compared to placebo. The analysis revealed an upregulation of cortisol and pregnenolone sulfate four hours after MDMA intake suggesting increased stress and serotonergic activity. Further, calcitriol levels were decreased after the intake of MDMA. Calcitriol is involved in the upregulation of trophic factors which have protective effects on brain dopamine neurons. The inflammation mediators hydroxyeicosatetraenoic acid (HETE), dihydroxyeicosatetraenoic acid (diHETE) and octadecadienoic acid (oxoODE) were found to be upregulated after the intake of MDMA compared to placebo which suggested a stimulation of inflammation pathways.
\end{abstract}




\section{INTRODUCTION}

3,4-Methylenedioxymethamphetamine (MDMA; 'ecstasy') is a popular recreational drug. It acts as indirect monoaminergic agonist releasing serotonin (5-hydroxytryptamine; 5-HT), norepinephrine (NE) and dopamine (DA) by interacting with the respective membrane transporters [Hysek, 2012 \#1243]. Little is known about peripheral and systemic changes on the level of endogenous metabolites caused by MDMA. MDMA increases glucocorticoids[Seibert, 2014 \#3228], prolactin , oxytocin, and arginine vasopressin in line with its predominant action to increase 5-HT [Seibert, 2014 \#3228;Hysek, 2012 \#1243;Hysek, 2014 \#2475;Kirkpatrick, 2014 \#5009;Simmler, 2011 \#1172]. However, metabolic changes on a wider spectrum are unstudied. Additionally, MDMA can cause hyperthermia, cardiac, hepatic and immune changes ${ }^{11,12}$ [Pacifici, 2004 \#288;Bigler, 2015 \#4488]. Only a few studies have illustrated the effect of MDMA consumption on inflammation pathways. It activates microglia cells in the brain triggering the secretion of proinflammatory cytokines, nitric oxide, prostaglandins and other reactive species which promote neuroinflammation processes and can damage neuronal tissue ${ }^{13,14}$.

Metabolomics screening approaches are suitable tools for the high-throughput identification of many metabolites within one single sample. At best, it can reveal several pathways which may play an important role in drug consumption. In a first attempt looking only at a limited number of endogenous metabolites, we could show that a single intake of MDMA showed changes in certain amino acids (AA) and biogenic amines that are rather time-dependent than influenced by MDMA. The metabolic ratio methionine-sulfoxide over methionine (MetSO/Met) was increased after the intake of MDMA indicating an increase of systemic oxidative stress. Further, an increase of diacyl-phosphatidyl-cholines and acyl-alkyl phosphatidylcholines after the intake of MDMA was observed which can be interpreted as an upregulation in energy production and changes in cell functions and inflammation processes ${ }^{15}$. Nielsen et al. retrospectively investigated forensic whole blood samples of humans exposed to MDMA with an untargeted screening approach and found among other metabolites changes of several acylcarnitines, lysophospatidylcholines (lysoPC), adenosine and inosine in response to MDMA 16 .

The human metabolome responds quite sensitive on many different influences such as food intake, stress, xenobiotics and physical exercise ${ }^{17,18}$. In this follow-up study, we aimed to find a broader spectrum of endogenous pathways and metabolites which could be involved or affected after MDMA consumption. Therefore, the aim of this study was to extensively investigate changes of plasma metabolites in response to a single dose of $125 \mathrm{mg}$ of MDMA. Samples from a placebo-controlled, double-blind crossover study [Schmid, 2015 \#3774] were analyzed with an untargeted metabolomics screening method to cover as many endogenous metabolites as possible.

\section{METHODS AND MATERIALS}

\section{Clinical Study}

Sample collection and details of the study have been published previously ${ }^{15,19}$. The samples were stored at our institute $\left(-80^{\circ} \mathrm{C}\right)$. In brief, eight male and eight female subjects (age 20-27) were recruited. Drug use history of subjects and study inclusion criteria has been described in detail elsewhere ${ }^{19,20}$. For the present analysis, only plasma samples from the session days where the participants took placebo or MDMA alone were used while other treatments were also administered in the study but not analyzed here. Wash-out periods between session days were at least 10 days. On the session days, MDMA (125 mg p.o., corresponding to $1.8 \pm 0.2 \mathrm{mg} / \mathrm{kg}$ body weight) or placebo was ingested at 10 a.m. Plasma samples used for this study were 
collected at 8 a.m. (= time point 0$), 2$ p.m. (= time point 1$), 6$ p.m.(= time point 2$)$, and the next day at 8 a.m. (= time point 3 ). A standardized meal was served at 12:30 p.m. during the session days. The clinical study was conducted at the University Hospital of Basel. The study was approved by the Ethics Committee of the Canton Basel, Switzerland and the Swiss Agency for Therapeutic Products (Swissmedics) and it was registered at ClinicalTrials.gov (NCT01771874). All subjects provided written informed consent and were paid for their participation.

\section{Chemicals and Reagents}

1-Methylhistidine, adenine, adenosine, arginine (arg), azelaic acid, chenodeoxycholic acid, cholic acid, citrulline, cortisol, cortisone, creatinine, deoxycholic acid, glutaric acid, glycocholic acid, hippuric acid, inosine, isoleucine (ile), leucine (leu), L-pyroglutamic acid, methionine (met), methylmalonic acid, mevalonolactone, N,N-dimethylglycine, nicotinic acid, p-aminobenzoic acid, phenylalanine (phe), proline (pro), raffinose, riboflavin, taurine, taurocholic acid, tryptophan, and uracil were purchased from Sigma-Aldrich (Buchs, Switzerland). Deuterated and heavy-labeled internal standards (IS) adenosine ribose-D1, arginine-13C6, caffeine 3-methyl-13C, carnitine trimethyl-D9, creatinine N-methyl-D3, deoxycholic acid-D4, D-fructose 13C, glycine-13C2, glycocholic acid-D4, hippuric acid 15N, kynurenine-D4, leucine-D10, lysine-D4, phenylalanine-D1, proline $15 \mathrm{~N}$, serine-D3, tryptophan-D5 and uric acid-15N2 were purchased from Cambridge isotope laboratories which were delivered by ReseaChem Life Science (Burgdorf, Switzerland) or Sigma-Aldrich (Buchs, Switzerland). Water, acetonitrile (ACN), methanol (MeOH) of HPLC grade were obtained from Fluka (Buchs, Switzerland). All other chemicals used were from Merck (Zug, Switzerland) and of the highest grade available.

\section{Sample preparation}

$150 \mu 1$ of plasma and $30 \mu \mathrm{l}$ of the IS mixture (table S-1,supporting information) were added to a tube. Protein precipitation was performed by addition of $450 \mu \mathrm{l}$ cooled $\mathrm{MeOH} /$ acetone $(90: 10 \mathrm{v} / \mathrm{v})$. The mixture was vortexed, centrifuged (10 min, 14'000 x g) and $250 \mu \mathrm{l}$ of the supernatant was transferred into two filter vials $(0.45 \mu \mathrm{m}$ PTFE, Thomson Instrument company, California, USA). Samples were stored at $-20{ }^{\circ} \mathrm{C}$ until measurements on the UHPLC-Q TOF as described below. Additionally, pooled samples with $10 \mu \mathrm{l}$ from every sample were prepared in the same manner and supernatant was aliquoted into six filter vials.

\section{UHPLC-Q TOF MS}

In total 128 samples (16 participants, 2 sessions, 4 time points) were subjected to mass spectrometric measurements. Samples were divided in two batches and a randomized analysis was performed on a Thermo Fischer Ultimate 3000 UHPLC system (Thermo Fischer Scientific, San Jose, CA) coupled to a high-resolution TOF instrument system (TripleTOF 6600, Sciex, Concord, Ontario, Canada). Mobile phases A and B consisted of $10 \mathrm{mM}$ ammonium formate with $0.1 \%(\mathrm{v} / \mathrm{v})$ formic acid in water and $0.1 \%(\mathrm{v} / \mathrm{v})$ formic acid in $\mathrm{MeOH}$, respectively. Mobile phases $\mathrm{C}$ and $\mathrm{D}$ were $25 \mathrm{mM}$ ammonium acetate and $0.1 \%(\mathrm{v} / \mathrm{v})$ acetic acid in water and $0.1 \%(\mathrm{v} / \mathrm{v})$ acetic acid in ACN, respectively.

Two different columns were used for chromatographic separation. A Waters (BadenDaettwil, Switzerland) XSelect HSST RP-C18 column $(150 \mathrm{~mm}$ x $2.1 \mathrm{~mm}, 2.5 \mu \mathrm{m}$ particle size) was applied with the following gradient: 1 min $100 \% \mathrm{~A} ; 1-15 \min 100 \% \mathrm{~B} ; 15-18 \mathrm{~min}$ $100 \%$ and then decreased to start conditions and re-equilibration for $2 \mathrm{~min}$. Flow rate increased after $15 \mathrm{~min}$ to $0.7 \mathrm{ml} / \mathrm{min}$. Further a Merck SeQuant ZIC HILIC column (150 mm x $2.1 \mathrm{~mm}$, $3.5 \mu \mathrm{m}$ particle size) using the following gradient: $1 \mathrm{~min} 95 \% \mathrm{D} ; 1-10 \mathrm{~min}$ decrease to $40 \%$ $\mathrm{D} ; 10-12 \min 10$ decrease to $10 \% \mathrm{D} ; 12-13 \min 10 \% \mathrm{D}$; and then increase to start conditions 
and re-equilibration for 2 min was used. The column oven was set to $40{ }^{\circ} \mathrm{C}$ and injection volume was $1 \mu \mathrm{l}$ for all samples.

High resolution mass spectra (MS) and MS/MS data were acquired by the two methods: TOF MS only and information dependent data acquisition (IDA) in positive and negative ionization mode. MS analysis was performed with a DuoSpray ion source at a resolving power (full width at half-maximum at m/z 400) of 30'000 in MS and 30'000 in MS2 (high-resolution mode) or $15^{\prime} 000$ (high-sensitivity mode) in positive ionization mode. Automatic calibration was obtained every three sample injections using atmospheric-pressure chemical ionization (APCI) positive calibration solution (Sciex) in the positive ionization mode and every five sample injections using APCI negative calibration solution (Sciex) in the negative ionization mode. The TOF MS method was composed of a TOF-MS scan over a mass range from m/z 50 to $\mathrm{m} / \mathrm{z} 1000$ (accumulation time $100 \mathrm{msec}, \mathrm{CE} 5 \mathrm{eV}$ ). Additionally, $20 \%$ of the samples were measured in the IDA scan mode. The IDA method consisted of a TOF-MS scan over a mass range from $\mathrm{m} / \mathrm{z}$ 50 to $\mathrm{m} / \mathrm{z} 1000$ (accumulation time $50 \mathrm{msec}$, CE $5 \mathrm{eV}$ ). IDA experiments (accumulation time for each IDA experiment $100 \mathrm{msec}, \mathrm{CE} 35 \mathrm{eV}$ with a CE spread of $15 \mathrm{eV}$ ) were performed after dynamic background subtraction on the four most intense ions with an intensity threshold above $100 \mathrm{cps}$ and exclusion time of $5 \mathrm{~s}$ (half peak width) after two occurrences in high sensitivity mode.

A system suitability test (SST) containing 1-methylhistidine, adenine, adenosine, arg, azelaic acid, chenodeoxycholic acid, cholic acid, citrulline, cortisol, cortisone, creatinine, deoxycholic acid, glutaric acid, glycocholic acid, hippuric acid, inosine, ile, leu, L-pyroglutamic acid, met, methylmalonic acid, mevalonolactone, N,N-dimethylglycine, nicotinic acid, p-aminobenzoic acid, phe, pro, raffinose, riboflavin, taurine, taurocholic acid, tryptophan and uracil (concentration $10 \mu \mathrm{g} / \mathrm{ml}$ each) was measured after every fifth sample. The SST was checked for reproducibility of the data by retention time (RT) shifts and peak area comparison using MultiQuant V 2.1 (Sciex). Further, a pooled sample was additionally measured after every fifth sample.

\section{Data analysis and evaluation}

Progenesis QI (Waters Corp, Milford, USA) was used for data-preprocessing, alignment, deconvolution, peak-picking, identification and simple statistical calculations. Automatic processes were checked manually and pooled plasma samples were used as reference samples for data alignment. Data files of the IDA scan mode were incorporated in the software for identification purposes only to have MS/MS spectra of the most abundant compounds. Identification was performed by searching against an in-house library in PeakView V 2.2 (Sciex) and against different online databases including METLIN ${ }^{21}$, the Human Metabolome Database $^{22}$ (HMDB, V4.0), NIST ${ }^{23}$ and Lipidblast ${ }^{24}$ using Progenesis QI. The in-house database was further converted into MSP files and incorporated in Progenesis QI.

Final identification results were classified on the different levels of identification confidence suggested by the metabolomics standard initiative (MSI) ${ }^{25}$ : Level 1, highest confidence based on matching exact mass, RT and fragmentation pattern with an in-house spectral library of standards collected under the same conditions. Level 2 requires spectrum and mass similarities to data available in public databases with reasonable RT. Level 3 assigns chemical classes based on spectral data and exact mass information relating on available public databases without retention time. Level 4 are unknown compounds.

\section{Statistical evaluation}

Statistical calculations were done with Prism 7 (GraphPad Software, CA, USA) and MetaboAnalyst $4.0^{26}$. Normalization was conducted in Progenesis QI. Progenesis calculates a factor which will be multiplied by all compound ion abundances for each sample, to allow recalibration to a normalization reference run. A quantitative abundance ratio of all detected 
ions was calculated between the normalized run and the pooled sample that was selected as reference sample.

Prior to statistical calculations, a missing value imputation was conducted with the MetImp online tool ${ }^{27}$. Random forest (RF) was chosen as imputation method for missing values ${ }^{27,28}$. Two-way repeated measure analysis of variance (ANOVA) (within subject) was calculated for time series analysis with application of Tukey test for multiple comparison. Paired t-tests were calculated separately on every time point to identify statistically significant $(\mathrm{p} \leq 0.01)$ differences between the two sessions.

Partial least square discriminant analysis (PLS-DA) was applied on autoscaled data of important metabolites including MDMA and MDMA-metabolites from timepoint 1. Cross model validation and permutating testing for validation of the PLS-DA model was used.

Baseline correction according to the first study was conducted for analytes, which showed a significant change (paired t-test, $\mathrm{p} \leq 0.05$ ) between time point 0 in the MDMA and placebo session ${ }^{15}$. The peak area of the analyte measured at time point 0 was subtracted from the peak area of the analyte at the other time points to overcome interday variabilities.

\section{RESULTS AND DISCUSSION}

\section{Instrument performance}

Analytes in the SST samples were normalized over the summarized intensities of all analytes to statistically equilibrate the instrument performance. Peak areas were calculated using Multiquant V2.1 (Sciex). The applied screening method has been extensively examined recently on analyte selection, detectability and sensitivity (unpublished work, Zurich Institute of Forensic Medicine, Switzerland, M.I. Boxler, T.D. Schneider, T. Kraemer, A.E. Steuer, May 2018). Measurements on the HSST column showed constant peak areas of the analytes and were highly reproducible. Chenodeoxycholic acid and deoxycholic acid showed a decreasing trend which can be explained by substance instabilities. Uric acid showed unexpected fluctuations in all experiments due to non-constant chromatographic retention time of this substance. Analytes in the SST samples which were measured on the HILIC column showed a slight retention time shift and overall, the pressure during the runs was not as stable as for the measurements on the HSST column. Overall, all experiments were reproducible in terms of peak intensity and retention time continuity and were therefore used for the analysis of the data.

\section{Normalization}

Normalization techniques have to deal with technical variation which can bias the scaling factor. Normalization process of Progenesis QI was compared to normalization processes via total ion count (TIC). Both reduced the coefficient of variation (CV) well (data not shown) and CV's were within the same range. However, to reduce overall calculations, the normalization process from Progenesis QI was finally selected and applied. Normalization processes via bestmatch IS did lead to inferior results compared to the other two methods and was excluded as normalization method. Additionally, two samples from different time points were missing and missing value imputation was conducted.

\section{Baseline correction}

The multilevel structure of the dataset is quite complex and a good statistical strategy is necessary. In the first project, baseline correction was conducted on all metabolites and analytes were selected as statistically significant if the p-value was $\leq 0.01^{15}$. With the baseline correction one can overcome interday variabilities, however it also removes drug-dependent changes in certain metabolites which are only little but could still be influenced by the intake of MDMA. For this extensive appraisal, baseline correction was only applied on certain metabolites. Peak areas of the identified metabolites were compared between the MDMA and placebo session at time point 0 and if there was a significant change ( $\mathrm{p} \leq 0.05$, paired $\mathrm{t}$-test) between the two 
samples, a baseline correction was conducted. If there was no significant difference visible in the two samples, metabolites were compared without a baseline correction (table 1).

\section{Detection of MDMA and its metabolites}

An unambiguous identification of MDMA and its main metabolites could be achieved using accurate masses and retention times. The highly significant p-values, fold changes, congruent fragments in the MS/MS fragmentation spectra and the compound absence in the placebo conditions further verified the findings. MDMA itself was identified only in the positive mode of both column settings. Since MDMA and its metabolites carry a basic nitrogen group, negative ionization is unlikely. Furthermore 3,4-methylenedioxyampehtamine (MDA), 3,4dihydroxymethamphetamine sulfates (DHMA 3-sulfate and DHMA 4-sulfate respectively), 4hydroxy-3-methoxymethamphetamine sulfate (HMMA sulfate), HMMA glucuronide and an in-source fragment of MDMA could be identified either with HSST or HILIC in both ionization modes. A PLS-DA analysis was conducted with MDMA, MDMA-metabolites and metabolites which were identified and statistically significant between the two sessions. A separation of the MDMA and the placebo group could be achieved (first component $R^{2}=0.83, Q^{2}=0.78$ ), with MDMA and its metabolites showing the highest variable importance parameters (VIPs) scores and therefore were primarily responsible for the group separation. The PLS-DA plot was validated and the $\mathrm{p}$-value ( $\mathrm{p}<0.001)$ of the prediction errors from 1000 randomly permutated data sets revealed a valid model with no overfit (figure 1). The separation of the two groups with MDMA and its metabolites having the highest impact was predictable and showed, that the applied screening method operated well.

\section{Identified endogenous metabolites}

With all four chromatographic modes (HSST positive and negative, HILIC positive and negative) around 36'000 compound ions were found, with the majority of the compound ions found in the positive ionization mode. After data processing with the data from the IDA mode, 10 ' 400 features were detected with an MS/MS spectrum. These features were further processed and statistically evaluated with fold change analysis. Time points were viewed separately and features with a fold change 2 or higher were selected for metabolite identification.

A total of 28 metabolites with an identification level of 2 or higher were detected, which showed a significant change $(\mathrm{p} \leq 0.01)$ between the MDMA and placebo session and/or the different time points (table 1). These metabolites do not include MDMA and MDMA metabolites. Further 27 metabolites and features respectively with an identification level of 3 or 4 , fold change analysis $>2$ and significant changes ( $\mathrm{p} \leq 0.05$, repeated measured ANOVA) between the MDMA and placebo session were detected with Progenesis Qi (table S-2, supporting information). Different lipids were identified, a diacylglycerid (DG(x:y)), lysophosphatidylcholines (lysoPCx:y), lysophosphatidylethanolamines (lysoPEx:y/x:y), glycerophosphatidylcholines (GPChox:y/x:y) and glycerophosphatidylethanolamine (GPEtnx:y/x:y). The side chains are indicated with " $x: y$ " where ' $x$ ' describes the number of carbons and ' $y$ ' the number of double bonds. The identified metabolites are listed in table 1. In the discussion, we mainly focused on alterations of steroid and inflammation pathways.

\section{Effects of MDMA intake}

In the previous publication, we stated that certain AA such as alanine, or tryptophan as well as other metabolites like taurine, creatinine and kynurenine underwent time-dependent changes independent from the MDMA intake. The metabolic ratio Met-SO/Met was increased after the intake of MDMA due to formation of reactive oxygen species after MDMA consumption. Further, an increase of fatty acids after the intake of MDMA was explained with a higher energy demand and production due to increased activity and cardiac stress ${ }^{15}$. Here in the second study, we only statistically analyzed features respectively metabolites, which showed a fold change 
greater than two between the placebo and the MDMA session. This statistical test omitted metabolites that showed only time-dependent changes. With this untargeted screening method, tryptophan showed statistically significant changes between the time points, but since we did not apply baseline correction on every metabolite, tryptophan showed also a statistically significant downregulation after MDMA intake compared to placebo at time point 1 (paired ttest, $\mathrm{p}=0.01$ ) which is congruent to the findings of Nielsen et al. ${ }^{16}$. Tryptophan is an important AA for the synthesis of 5-HT which is suggested to be upregulated after MDMA consumption 11,29 .

\section{Steroid metabolism}

Focusing on the steroid metabolism, three altered metabolites could be identified and characterized. Cortisol was unambiguously identified and showed highly significant changes at time point 1 ( $\mathrm{p}=0.0003)$. Compared to the intake of placebo, cortisol levels increased after the intake of MDMA before lowering back down on the same level as after placebo consumption (figure 2). These findings were already described in targeted studies on cortisol changes based on MDMA intake ${ }^{30-32}$. Cortisol is a glucocorticoid steroid which is released in response to stress or low blood-glucose concentration and it is also a marker for serotonergic activity ${ }^{33}$. MDMA is further known to increase the secretion of prolactin, another serotonergic activity marker, and noradrenaline (NA), which plasma levels represent mostly an overflow of NA from the sympathetic nerves ${ }^{30}$.

Moreover, pregnenolone sulfate (PregS) was elevated at time point 1 and 2 ( $p=0.0003$ and $\mathrm{p}=0.043$ respectively). It is a neuroactive steroid that is synthesized from pregnelonone (Preg)

${ }^{34}$. Cholesterol is converted into Preg which is the first step in the steroid-synthesis. Preg can further undergo various metabolic pathways and is mainly converted to PregS, progesterone (Prog) and 17-hydroxypregnenolone in humans ${ }^{35}$. Harris et al. investigated effects of a single intake of MDMA on various hormones including progesterone in the female participants $(\mathrm{n}=$ 3). They reported a progesterone increase after the intake of MDMA but the changes were not statistically significant compared to a placebo intake ${ }^{36}$. Progesterone can be metabolized to 17hydroxyprogesterone which is further metabolized to glucocorticoids, mainly cortisol, or to androstenediones. In our study, the active steroid PregS showed a similar abundance profile as cortisol which supports the connected behavior. PregS regulates many brain functions by binding to $\gamma$-aminobutyric acid $\left(\mathrm{GABA}_{\mathrm{A}}\right)$ and $\mathrm{N}$-methyl-D-aspartate (NMDA) type receptors with high affinity ${ }^{35}$. Le Mélledo et. al. showed, that plasma PregS levels were significantly reduced in male patients with generalized anxiety disorders or with generalized social phobia compared to healthy male subjects ${ }^{37,38}$.

Calcitriol, the active metabolite of vitamin $\mathrm{D}_{3}$, showed a highly significant decrease at time point $1(p=0.0002)$ after the intake of MDMA compared to the placebo intake (figure 2$)$. This metabolite has potent effects on brain cells and may appear to upregulate trophic factors including the glial cell line-derived neurotrophic factor (GDNF) which has protective effect on brain DA neurons ${ }^{39}$. Cass et al. showed, that a treatment with calcitriol can protect against dopamine and serotonin depleting effects of neurotoxic doses of methamphetamine in rats. Animals which were treated with calcitriol showed significantly less methamphetamine reductions in DA, 5-HT and metabolites compared to the animals which were treated with placebo ${ }^{40}$. Methamphetamine and MDMA share structure similarities with similar neurotoxic effects ${ }^{2}$. Although these discussed metabolites can cross the blood brain barrier, the relevance of plasma levels to central nervous system activity is difficult to determine ${ }^{40,41}$. A complete and targeted analysis focusing on these pathways only would possibly reveal further connections.

\section{Inflammation pathways}


As already discussed in the first publication, reactive quinone-metabolites of MDMA can produce semiquinone radicals causing the generation of reactive oxygen species (ROS) which can lead to systemic oxidative stress and inflammation processes ${ }^{42,43}$. The three putatively identified metabolites, a hydroxyl-eicosatetraenoic acid (HETE) derivative, dihydroxyeicosatetraenoic acid (diHETE) and an oxo-octadecanedienoic acid (oxoODE) are inflammation mediators. The positions of the functional groups were not completely determinable based on the acquired measurements. With referral to the fragmentation data the compounds might be 5-HETE, 5,12-diHETE, and 13-oxoODE. These three metabolites showed similar trends over the analyzed time points with significantly increased peak areas after the intake of MDMA compared to placebo (figure 3). 12-diHETE was baseline corrected. Anneken et al described that there are multiple signaling pathways through which MDMA may increase cyclooxygenase (COX) activity and the subsequent production of prostaglandins ${ }^{44}$. Polyunsaturated fatty acids such as linoleic acid (LA) and arachidonic acid are oxidized to fatty acid hydroperoxides. In a first step, LA is oxidized to the peroxide hydroperoxyoctadecanedinoic acid (HPODE) which is then reduced to 9- and 13-HODE and further dehydrogenated to oxoODE. Similarly, arachidonic acid is oxidized to hydroperoxyeicosatetraenoic acid (HPETE) and reduced to HETE. A product of HETE is diHETE via an oxidation and reduction step. These arachidonic acid products which are isomers of leukotriene B4, were discussed as pro-inflammatory agents ${ }^{45,46}$. 13-oxoODE has also antiinflammatory effects ${ }^{47}$. For a broad analysis of these inflammation mediators in dependence of a MDMA intake, more metabolites from these involved pathways need to be investigated but these findings may serve as first valuable indicators.

Neurotransmitters such as DA and 5-HT, which play an important role in the pharmacology of MDMA, were not analyzed with the screening method. A targeted search for these metabolites was not conducted since plasma levels of these neurotransmitters can't be correlated to levels in the brain without special sample preparation and handling of the plasma 48

\section{CONCLUSION}

The metabolites involved in steroid and inflammation pathways which could be identified showed statistically significant changes after a single intake of MDMA compared to placebo, indicating increased stress, serotonergic activity and inflammation processed in the human body. With these results, we were able to confirm findings from a previous study on only a limited number of metabolites and were able to show additional markers mainly from steroidor inflammation pathways. . Untargeted metabolomics screening methods are a useful tool to get an insight in pathways which might be involved in the pharmacology and toxicology of a drug of abuse such as MDMA. However, as these pathways are quite complex and many endogenous metabolites are involved, further selective analysis of the individual pathways which showed to be altered after consumption would be necessary to obtain deeper insights into the underlying mechanisms. 


\section{FIGURES}

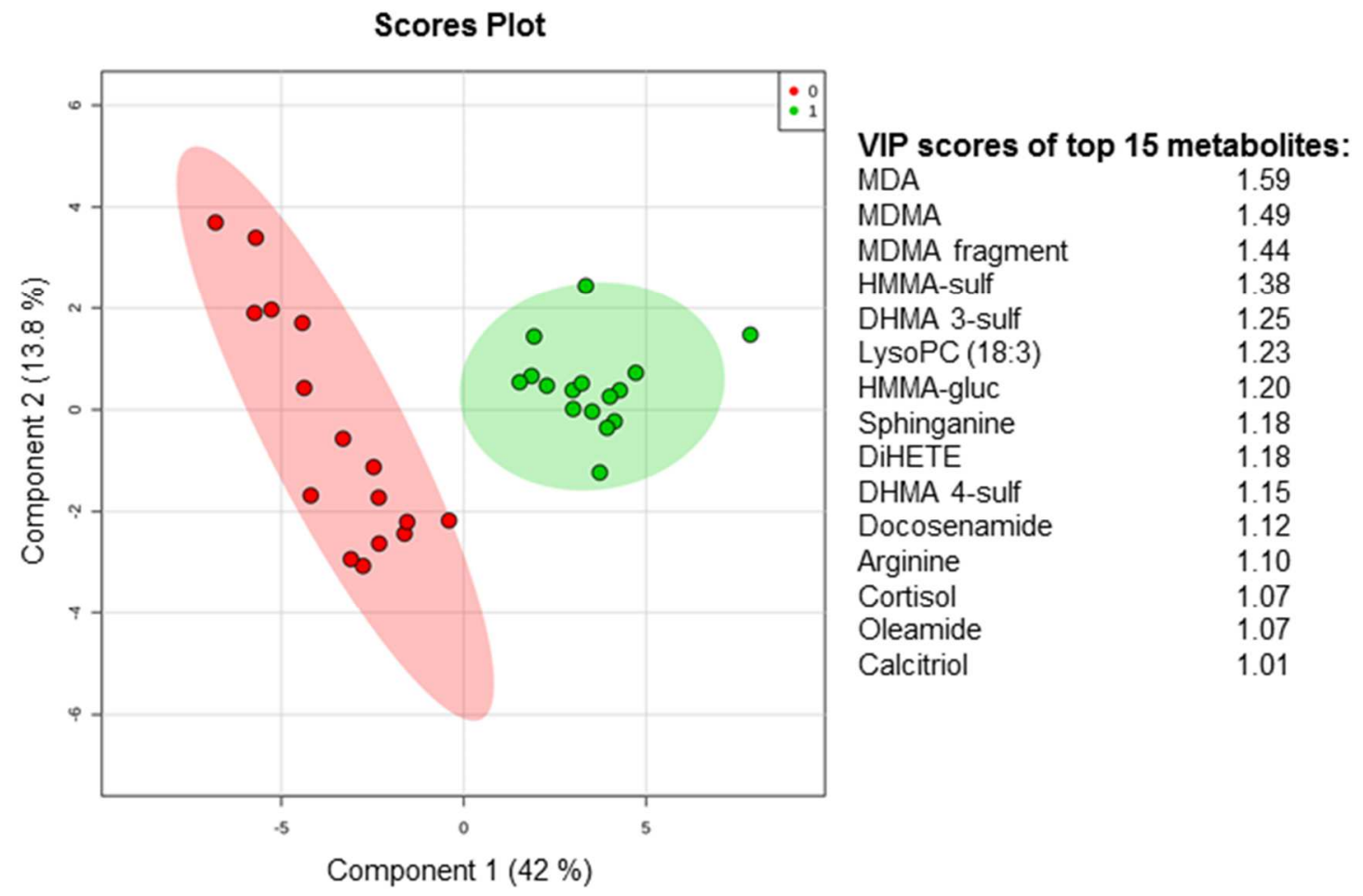

Figure 1. PLS-DA scores plot at timepoint 1. MDMA (green) or placebo (red) intake of 16 participants and resulting 95\% confidence region. Further VIP scores of component 1 of top 15 metabolites are listed. 

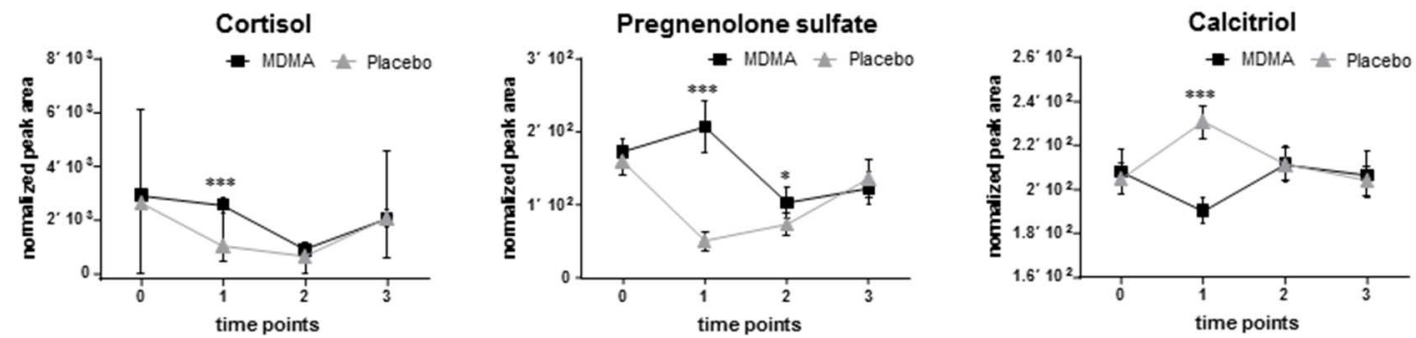

Figure 2. Metabolites of the steroid family which showed significant differences between MDMA and placebo intake. Squares represent the MDMA session and triangles represent the placebo session. Mean values with standard error measurements (SEM). Significance level is indicated with asterisks $(*=0.05-0.01 ; * *=0.01-0.001 ; * * *=0.001-0.0001)$ 

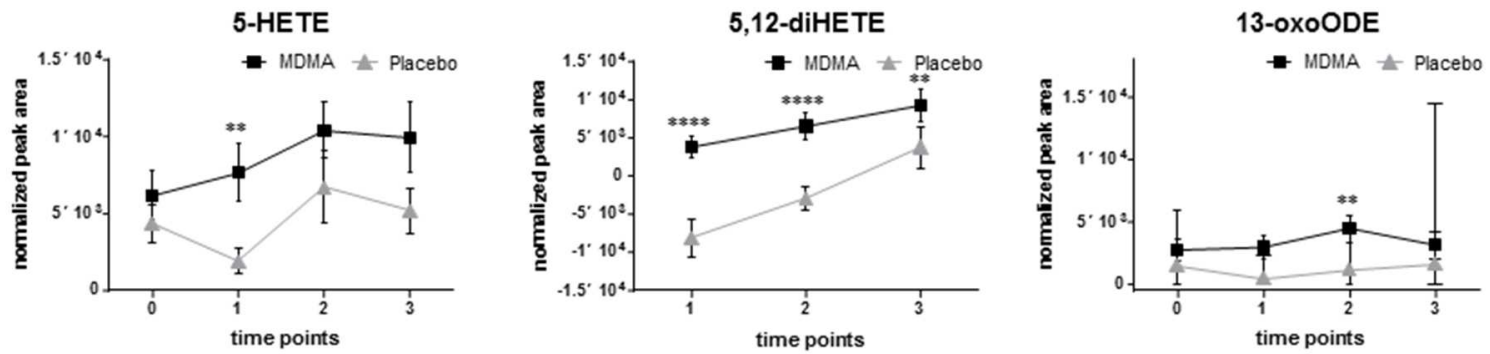

Figure 3. Inflammation mediators, squares represent the MDMA session and triangles the placebo session. Mean values with standard error measurements (SEM). Significance level is indicated with asterisks $(*=0.05-0.01 ; * *=0.01-0.001 ; * * *=0.001-0.0001)$ 
TABLES.

Table 1. Identified metabolites which showed a significant change between the MDMA and placebo session, arranged in compound classes

\begin{tabular}{|c|c|c|c|c|c|c|c|c|c|c|c|c|}
\hline$\underset{\mathscr{E}}{\stackrel{\mathscr{Z}}{Z}}$ & 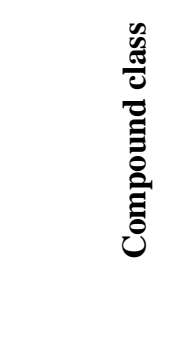 & $\stackrel{\mathbb{\Xi}}{ }$ & $\frac{\bar{g}}{\tilde{Z}}$ & 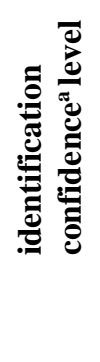 & 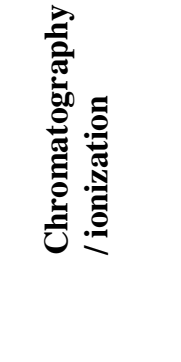 & 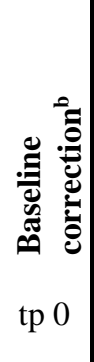 & $\begin{array}{l}\text { Time- } \\
\text { points }\end{array}$ & 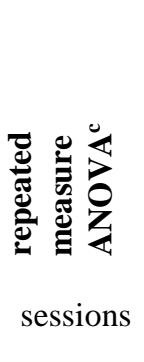 & $\begin{array}{l}\text { Inter- } \\
\text { action }\end{array}$ & tp 1 & 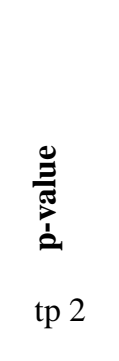 & tp 3 \\
\hline Spinganine & Amine & 302.304 & {$[\mathrm{M}+\mathrm{H}]^{+}$} & 2 & HSST pos. & n.s. & n.s. & $* *$ & $*$ & 0.0001 & n.s. & n.s. \\
\hline Arginine & Amino acid & 175.1189 & {$[\mathrm{M}+\mathrm{H}]^{+}$} & 1 & HSST pos. & n.s. & n.s. & $* *$ & n.s. & 0.0002 & n.s. & n.s. \\
\hline Glutamine & Amino acid & 147.0758 & {$[\mathrm{M}+\mathrm{H}]^{+}$} & 1 & HSST pos. & n.s. & n.s. & $*$ & n.s. & 0.0052 & n.s. & n.s. \\
\hline Histidine & Amino acid & 156.0764 & {$[\mathrm{M}+\mathrm{H}]^{+}$} & 1 & HSST pos. & n.s. & n.s. & $*$ & n.s. & 0.0012 & n.s. & n.s. \\
\hline Methionine & Amino acid & 150.0567 & {$[\mathrm{M}+\mathrm{H}]^{+}$} & 1 & HILIC pos. & n.s. & n.s. & $* * * *$ & n.s. & 0.0014 & 0.0046 & n.s. \\
\hline Tryptophan & Amino acid & 205.0967 & {$[\mathrm{M}+\mathrm{H}]^{+}$} & 1 & HSST pos. & n.s. & $* *$ & $*$ & n.s. & 0.0098 & n.s. & n.s. \\
\hline Tyrosine & Amino acid & 180.0666 & {$[\mathrm{M}-\mathrm{H}]^{-}$} & 1 & HILIC neg. & n.s. & $*$ & $* * *$ & $*$ & 0.0001 & 0.002 & n.s. \\
\hline Biliverdin & Bilirubin & 583.2543 & {$[\mathrm{M}+\mathrm{H}]^{+}$} & 2 & HILIC pos. & n.s. & $*$ & $* *$ & $*$ & 0.0022 & n.s. & n.s. \\
\hline Carnitine & Carnitine & $\begin{array}{c}162.1123 \\
184.092\end{array}$ & $\begin{array}{c}{[\mathrm{M}+\mathrm{H}]^{+}} \\
{[\mathrm{M}+\mathrm{Na}]^{+}}\end{array}$ & 1 & HSST pos. & n.s. & n.s. & $*$ & n.s. & 0.0016 & n.s. & n.s. \\
\hline Propionylcarnitine & Carnitine & 218.1381 & {$[\mathrm{M}+\mathrm{H}]^{+}$} & 1 & HSST pos. & n.s. & $* *$ & $* *$ & n.s. & 0.0043 & n.s. & n.s. \\
\hline
\end{tabular}




\begin{tabular}{|c|c|c|c|c|c|c|c|c|c|c|c|c|}
\hline $\begin{array}{l}\text { Hydroxyeicosatetraenoic } \\
\text { acid }\end{array}$ & Eicosanoid & 319.2288 & {$[\mathrm{M}-\mathrm{H}]^{-}$} & 2 & HILIC neg. & n.s. & n.s. & $* *$ & n.s. & 0.0097 & n.s. & n.s. \\
\hline Oleamide & Fatty acid & 282.2795 & {$[\mathrm{M}+\mathrm{H}]^{+}$} & 2 & HSST pos. & n.s. & n.s. & $*$ & n.s. & 0.0008 & n.s. & n.s. \\
\hline Docosenamide & Fatty amide & 338.3414 & {$[\mathrm{M}+\mathrm{H}]^{+}$} & 2 & HSST pos. & n.s. & n.s. & $*$ & n.s. & 0.0004 & n.s. & n.s. \\
\hline & & 360.3234 & {$[\mathrm{M}+\mathrm{Na}]^{+}$} & & & & & & & & & \\
\hline Hexadecanoyl glycerol & Glycerolipid & 331.283 & {$[\mathrm{M}+\mathrm{H}]^{+}$} & 2 & HSST pos. & n.s. & n.s. & $* *$ & $*$ & n.s. & n.s. & 0.0022 \\
\hline Glycerol stearate & Glycerolipid & 359.3157 & {$[\mathrm{M}+\mathrm{H}]^{+}$} & 2 & HSST pos. & n.s. & n.s. & $*$ & n.s. & n.s. & n.s. & 0.0035 \\
\hline & & 376.3423 & $\begin{array}{c}{\left[\mathrm{M}+\mathrm{NH}_{4}\right]} \\
+\end{array}$ & & & & & & & & & \\
\hline & & 381.2974 & {$[\mathrm{M}+\mathrm{Na}]^{+}$} & & & & & & & & & \\
\hline & & 341.305 & $\begin{array}{c}{[\mathrm{M}+\mathrm{H}-} \\
\left.\mathrm{H}_{2} \mathrm{O}\right]^{+}\end{array}$ & & & & & & & & & \\
\hline GPCho (16:0/18:2) & $\begin{array}{l}\text { Glycerophosphatid } \\
\text { yl-cholines }\end{array}$ & 758.5691 & {$[\mathrm{M}+\mathrm{H}]^{+}$} & 2 & HSST pos. & n.s. & n.s. & $* *$ & n.s. & 0.0027 & n.s. & 0.001 \\
\hline $\begin{array}{l}\text { GPCho } \quad(18: 2 / 18: 2) \\
(\operatorname{PC}(36: 4))\end{array}$ & $\begin{array}{l}\text { Glycerophosphatid } \\
\text { yl-cholines }\end{array}$ & 782.5679 & {$[\mathrm{M}+\mathrm{H}]^{+}$} & 2 & HSST pos. & n.s. & n.s. & $* * *$ & n.s. & n.s. & n.s. & $<0.0001$ \\
\hline $\begin{array}{l}\text { Dihydroxyeicosatetra- } \\
\text { enoic acid }\end{array}$ & Leukotriene & 335.2233 & {$[\mathrm{M}+\mathrm{H}]^{+}$} & 2 & HILIC neg. & $\mathrm{BC}$ & $* * *$ & $* * *$ & n.s. & $<0.00001$ & $<0.00001$ & 0.0037 \\
\hline Octadecadienoic acid & Linoelic acid & 293.2114 & {$[\mathrm{M}-\mathrm{H}]^{-}$} & 2 & HILIC neg. & n.s. & n.s. & $* *$ & n.s. & n.s. & 0.0083 & n.s. \\
\hline LysoPC (18:2) & $\begin{array}{l}\text { Lysophosphadityl- } \\
\text { choline }\end{array}$ & 520.3402 & {$[\mathrm{M}+\mathrm{H}]^{+}$} & 2 & HSST pos. & n.s. & n.s. & $* *$ & n.s. & n.s. & n.s. & 0.0034 \\
\hline LysoPC (18:3) & $\begin{array}{l}\text { Lysophosphadityl- } \\
\text { choline }\end{array}$ & 518.321 & {$[\mathrm{M}+\mathrm{H}]^{+}$} & 2 & HILIC pos. & n.s. & n.s. & $* * *$ & n.s. & $<0.0001$ & n.s. & n.s. \\
\hline
\end{tabular}




\begin{tabular}{|c|c|c|c|c|c|c|c|c|c|c|c|c|}
\hline LysoPC (22:6) & $\begin{array}{l}\text { Lysophosphatidyl- } \\
\text { choline }\end{array}$ & 568.3391 & {$[\mathrm{M}+\mathrm{H}]^{+}$} & 2 & HSST pos. & n.s. & n.s. & $* *$ & n.s. & 0.0065 & n.s. & 0.0091 \\
\hline LysoPE (18:0/0:0) & $\begin{array}{l}\text { Lysophosphatidyl- } \\
\text { ethanolamine }\end{array}$ & 480.3064 & {$[\mathrm{M}-\mathrm{H}]^{-}$} & 1 & HSST neg. & $\mathrm{BC}$ & n.s. & $* *$ & $*$ & 0.0002 & n.s. & n.s. \\
\hline LysoPE (18:2/0:0) & $\begin{array}{l}\text { Lysophosphatidy- } \\
\text { lethanolamine }\end{array}$ & 476.2791 & {$[\mathrm{M}-\mathrm{H}]^{-}$} & 2 & HILIC neg. & n.s. & $*$ & $* * *$ & n.s. & n.s. & 0.0009 & $<0.0001$ \\
\hline Calcitriol & Steroid & 399.3252 & $\begin{array}{l}\mathrm{M}+\mathrm{H}- \\
\mathrm{H} 2 \mathrm{O}\end{array}$ & 2 & HSST pos. & n.s. & n.s. & n.s. & n.s. & 0.0002 & n.s. & n.s. \\
\hline Cortisol & Steroid & 363.2147 & {$[\mathrm{M}+\mathrm{H}]^{+}$} & 1 & HSST pos. & n.s. & $* * * *$ & $* *$ & $* * *$ & 0.0003 & n.s. & n.s. \\
\hline Pregnenolone sulfate & Steroid & 395.1877 & {$[\mathrm{M}-\mathrm{H}]^{-}$} & 2 & HSST neg. & n.s. & $* * * *$ & $* * *$ & $* * * *$ & 0.0003 & n.s. & n.s. \\
\hline
\end{tabular}

${ }^{a}$ metabolites were identified based on different levels of identification confidence suggested by the metabolomics standard initiative

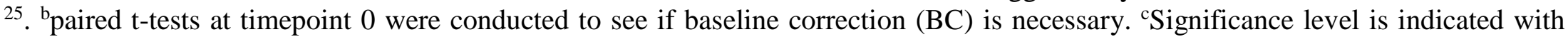
asteriks (n.s. = not significant; $*=0.05-0.01 ; * *=0.01-0.001 ; * * *=0.001-0.0001)$ 


\section{ASSOCIATED CONTENT}

Table S-1. Analytes and concentration of the internal standard mixture

Table S-2. Metabolites and features with an identification level 3 or 4 that significantly changed at one or more timepoints between the MDMA or placebo intake 


\section{AUTHOR INFORMATION}

\section{Corresponding Author}

Dr. Andrea E. Steuer

University of Zurich

Zurich Institute of Forensic Medicine (ZIFM)

Department of Forensic Pharmacology \& Toxicology

Winterthurerstrasse 190/52

CH-8057 Zurich

Switzerland

Tel.: 0041 446355679; fax: 0041446356852

E-mail address: andrea.steuer@irm.uzh.ch 


\section{Author Contributions}

The manuscript was written through contributions of all authors. All authors have given approval to the final version of the manuscript.

\section{Funding Sources}

Part of this study was supported by the Swiss National Science Foundation (grant no. 320030_1449493 and 320030_170249 to MEL).

\section{Notes}

The authors declare no competing financial interest.

\section{ACKNOWLEDGMENT}

The authors want to express their gratitude to Emma Louise Kessler, MD for her generous legacy she donated to the Institute of Forensic Medicine at the University of Zurich, Switzerland for research purposes.

\section{ABBREVIATIONS}

MDMA, 3,4-methylenedioxymethamphetamine; UHPLC-QTOF, high resolution quadrupole time of flight mass spectrometer coupled to liquid chromatography; HILIC, hydrophobic interaction liquid chromatography; HETE, hydroxyeicosatetraenoic acid; diHETE, dihydroxyeicosatetraenoic acid; oxoODE, octadecadienoic acid; DA, dopamine; 5-HT, 5-hydroxytryptamine; AA, amino acids; Met-SO/Met, methionine-sulfoxide over methionine; lysoPC, lysophospatidylcholines; Arg, arginine; ile, isoleucine; leu, leucine; met, methionine; phe, phenylalanine; pro, proline; IS, internal standard; CAN, acetonitrile; $\mathrm{MeOH}$, methanol; MS, mass spectra; IDA, information dependent data acquisition; APCI, atmospheric-pressure chemical ionization; SST, system suitability test; MSI, metabolomics standard initiative; RF, random forest; ANOVA, analysis of variance; PLS-DA, partial least square discriminant analysis; TIC, total ion count; CV, coefficient of variation; MDA, 3,4-methylenedioxyampehtamine; DHMA, 3,4-dihydroxymethamphetamine; HMMA, 4-hydroxy-3-methoxymethamphetamine; VIP, variable importance parameters; DG, diacylglycerid; lysoPE, lysophosphatidylethanolamines; GPCho, glycerophosphatidylcholines; GPEtn, glycerophosphatidylethanolamine; NA, noradrenaline; PregS, pregnenolone sulfate; Preg, pregnenolone; Prog, progesterone; GABA, $\gamma$-aminobutyric acid; NMDA, N-methyl-D-aspartate; GDNF, glia cell line-derived neurotrophic factor; COX, cyclooxygenase; LA, linoleic acid; HPODE, hydroperoxyoctadecanedinoic acid; HODE, hydroperoxyoctadecanedinoic acid; HPETE, hydroperoxyeicosatetraenoic acid 


\section{REFERENCES}

1

2 Moratalla, R. et al. Amphetamine-related drugs neurotoxicity in humans and in experimental animals: Main mechanisms. Progress in neurobiology 155, 149-170, doi:10.1016/j.pneurobio.2015.09.011 (2017).

3 Capela, J. P. et al. Molecular and cellular mechanisms of ecstasy-induced neurotoxicity: an overview. Molecular neurobiology 39, 210-271, doi:10.1007/s12035-009-8064-1 (2009).

4 Costa, G., Morelli, M. \& Simola, N. Repeated Administration of 3,4Methylenedioxymethamphetamine (MDMA) Elevates the Levels of Neuronal Nitric Oxide Synthase in the Nigrostriatal System: Possible Relevance to Neurotoxicity. Neurotoxicity research, doi:10.1007/s12640-018-9892-4 (2018).

5 Monks, T. J., Jones, D. C., Bai, F. \& Lau, S. S. The role of metabolism in 3,4-(+)methylenedioxyamphetamine and 3,4-(+)-methylenedioxymethamphetamine (ecstasy) toxicity. Ther Drug Monit 26, 132-136 (2004).

6 McCann, U. D. et al. Positron emission tomographic studies of brain dopamine and serotonin transporters in abstinent (+/-)3,4-methylenedioxymethamphetamine ("ecstasy") users: relationship to cognitive performance. Psychopharmacology 200, 439-450, doi:10.1007/s00213-008-1218-4 (2008).

7 Mueller, M. et al. Direct comparison of (+/-) 3,4-methylenedioxymethamphetamine ("ecstasy") disposition and metabolism in squirrel monkeys and humans. Ther Drug Monit 31, 367-373, doi:10.1097/FTD.0b013e3181a4f6c2 (2009).

8 Watson, R. Ecstasy is making a comeback, European drugs report warns. Bmj 353, i3078, doi:10.1136/bmj.i3078 (2016).

9 Poland, R. E. et al. Abnormal ACTH and prolactin responses to fenfluramine in rats exposed to single and multiple doses of MDMA. Psychopharmacology 131, 411-419 (1997).

10 Connor, T. J. et al. Acute 3,4-methylenediox ymethamphetamine(MDMA) administration produces a rapid and sustained suppression of immune function in the rat. Immunopharmacology 38, 253-260 (1998).

Liechti, M. E. Effects of MDMA on body temperature in humans. Temperature 1, 192200, doi:10.4161/23328940.2014.955433 (2014).

13 Yamamoto, B. K. \& Raudensky, J. The role of oxidative stress, metabolic compromise, and inflammation in neuronal injury produced by amphetamine-related drugs of abuse. Journal of neuroimmune pharmacology : 3, 203-217, doi:10.1007/s11481-008-9121-7 (2008).

14 Orio, L. et al. 3,4-Methylenedioxymethamphetamine increases interleukin-1 beta levels and activates microglia in rat brain: studies on the relationship with acute hyperthermia and 5HT depletion. Journal of neurochemistry 89, 1445-1453, doi:10.1111/j.14714159.2004.02443.x (2004).

15 Boxler, M. I., Liechti, M. E., Schmid, Y., Kraemer, T. \& Steuer, A. E. First Time View on Human Metabolome Changes after a Single Intake of 3,4Methylenedioxymethamphetamine in Healthy Placebo-Controlled Subjects. Journal of proteome research 16, 3310-3320, doi:10.1021/acs.jproteome.7b00294 (2017). 
16 Nielsen, K. L., Telving, R., Andreasen, M. F., Hasselstrom, J. B. \& Johannsen, M. A Metabolomics Study of Retrospective Forensic Data from Whole Blood Samples of Humans Exposed to 3,4-Methylenedioxymethamphetamine: A New Approach for Identifying Drug Metabolites and Changes in Metabolism Related to Drug Consumption. Journal of proteome research 15, 619-627, doi:10.1021/acs.jproteome.5b01023 (2016).

17 Dallmann, R., Viola, A. U., Tarokh, L., Cajochen, C. \& Brown, S. A. The human circadian metabolome. Proceedings of the National Academy of Sciences of the United States of America 109, 2625-2629, doi:10.1073/pnas.1114410109 (2012).

18 O'Sullivan, A., Gibney, M. J. \& Brennan, L. Dietary intake patterns are reflected in metabolomic profiles: potential role in dietary assessment studies. The American journal of clinical nutrition 93, 314-321, doi:10.3945/ajcn.110.000950 (2011).

19 Schmid, Y. et al. Interactions between bupropion and 3,4methylenedioxymethamphetamine in healthy subjects. The Journal of pharmacology and experimental therapeutics 353, 102-111, doi:10.1124/jpet.114.222356 (2015).

20 Steuer, A. E. et al. Chiral Plasma Pharmacokinetics of 3,4Methylenedioxymethamphetamine and its Phase I and II Metabolites following Controlled Administration to Humans. Drug metabolism and disposition: the biological fate of chemicals 43, 1864-1871, doi:10.1124/dmd.115.066340 (2015).

21 Guijas, C. et al. METLIN: A Technology Platform for Identifying Knowns and Unknowns. Analytical chemistry 90, 3156-3164, doi:10.1021/acs.analchem.7b04424 (2018).

22 Wishart, D. S. et al. HMDB: the Human Metabolome Database. Nucleic acids research 35, D521-526, doi:10.1093/nar/gk1923 (2007).

23 Linstrom, P. J. \& Mallard, W. G. NIST Chemistry WebBook; NIST Standard Reference Database No. 69. (2001).

24 Kind, T. et al. LipidBlast in silico tandem mass spectrometry database for lipid identification. Nature methods 10, 755-758, doi:10.1038/nmeth.2551 (2013).

25 Sumner, L. W. et al. Proposed minimum reporting standards for chemical analysis Chemical Analysis Working Group (CAWG) Metabolomics Standards Initiative (MSI). Metabolomics : Official journal of the Metabolomic Society 3, 211-221, doi:10.1007/s11306-007-0082-2 (2007).

26 Chong, J. et al. MetaboAnalyst 4.0: towards more transparent and integrative metabolomics analysis. Nucleic acids research, doi:10.1093/nar/gky310 (2018).

27 Wei, R. et al. Missing Value Imputation Approach for Mass Spectrometry-based Metabolomics Data. Scientific reports 8, 663, doi:10.1038/s41598-017-19120-0 (2018).

28 Gromski, P. S. et al. Influence of missing values substitutes on multivariate analysis of metabolomics data. Metabolites 4, 433-452, doi:10.3390/metabo4020433 (2014).

29 Boadle-Biber, M. C. Regulation of serotonin synthesis. Progress in biophysics and molecular biology 60, 1-15 (1993).

30 Hysek, C. M. et al. Pharmacokinetic and pharmacodynamic effects of methylphenidate and MDMA administered alone or in combination. The international journal of neuropsychopharmacology 17, 371-381, doi:10.1017/S1461145713001132 (2014).

31 Wolff, K. et al. Pharmacogenetic studies of change in cortisol on ecstasy (MDMA) consumption. Journal of psychopharmacology 26, 419-428, doi:10.1177/0269881111415737 (2012). 
32 Dumont, G. J. \& Verkes, R. J. A review of acute effects of 3,4methylenedioxymethamphetamine in healthy volunteers. Journal of psychopharmacology 20, 176-187, doi:10.1177/0269881106063271 (2006).

33 Lefebvre, H. et al. Serotonin-induced stimulation of cortisol secretion from human adrenocortical tissue is mediated through activation of a serotonin4 receptor subtype. Neuroscience 47, 999-1007 (1992).

34 Zorumski, C. F., Paul, S. M., Izumi, Y., Covey, D. F. \& Mennerick, S. Neurosteroids, stress and depression: potential therapeutic opportunities. Neuroscience and biobehavioral reviews 37, 109-122, doi:10.1016/j.neubiorev.2012.10.005 (2013).

35 Vallee, M. Neurosteroids and potential therapeutics: Focus on pregnenolone. The Journal of steroid biochemistry and molecular biology 160, 78-87, doi:10.1016/j.jsbmb.2015.09.030 (2016).

36 Harris, D. S., Baggott, M., Mendelson, J. H., Mendelson, J. E. \& Jones, R. T. Subjective and hormonal effects of 3,4-methylenedioxymethamphetamine (MDMA) in humans. Psychopharmacology 162, 396-405, doi:10.1007/s00213-002-1131-1 (2002).

37 Semeniuk, T., Jhangri, G. S. \& Le Melledo, J. M. Neuroactive steroid levels in patients with generalized anxiety disorder. The Journal of neuropsychiatry and clinical neurosciences 13, 396-398, doi:10.1176/jnp.13.3.396 (2001).

38 Heydari, B. \& Le Melledo, J. M. Low pregnenolone sulphate plasma concentrations in patients with generalized social phobia. Psychological medicine 32, 929-933 (2002).

39 Lin, L. F., Doherty, D. H., Lile, J. D., Bektesh, S. \& Collins, F. GDNF: a glial cell linederived neurotrophic factor for midbrain dopaminergic neurons. Science 260, 1130-1132 (1993).

40 Cass, W. A., Smith, M. P. \& Peters, L. E. Calcitriol protects against the dopamine- and serotonin-depleting effects of neurotoxic doses of methamphetamine. Annals of the New York Academy of Sciences 1074, 261-271, doi:10.1196/annals.1369.023 (2006).

41 Wang, M. D., Wahlstrom, G. \& Backstrom, T. The regional brain distribution of the neurosteroids pregnenolone and pregnenolone sulfate following intravenous infusion. The Journal of steroid biochemistry and molecular biology 62, 299-306 (1997).

42 Aruoma, O. I., Grootveld, M. \& Bahorun, T. Free radicals in biology and medicine: from inflammation to biotechnology. BioFactors 27, 1-3 (2006).

43 Quinton, M. S. \& Yamamoto, B. K. Causes and consequences of methamphetamine and MDMA toxicity. The AAPS journal 8, E337-347, doi:10.1208/aapsj080238 (2006).

44 Anneken, J. H., Cunningham, J. I., Collins, S. A., Yamamoto, B. K. \& Gudelsky, G. A. MDMA increases glutamate release and reduces parvalbumin-positive GABAergic cells in the dorsal hippocampus of the rat: role of cyclooxygenase. Journal of neuroimmune pharmacology : the official journal of the Society on NeuroImmune Pharmacology 8, 5865, doi:10.1007/s11481-012-9420-x (2013).

45 Kang, K. H., Liou, H. H., Hour, M. J., Liou, H. C. \& Fu, W. M. Protection of dopaminergic neurons by 5-lipoxygenase inhibitor. Neuropharmacology 73, 380-387, doi:10.1016/j.neuropharm.2013.06.014 (2013).

46 Bittleman, D. B. \& Casale, T. B. 5-Hydroxyeicosatetraenoic acid (HETE)-induced neutrophil transcellular migration is dependent upon enantiomeric structure. American journal of respiratory cell and molecular biology 12, 260-267, doi:10.1165/ajrcmb.12.3.7873191 (1995). 
47 Altmann, R. et al. 13-Oxo-ODE is an endogenous ligand for PPARgamma in human colonic epithelial cells. Biochemical pharmacology 74, 612-622, doi:10.1016/j.bcp.2007.05.027 (2007).

48 Yubero-Lahoz, S. et al. Effects of MDMA and related analogs on plasma 5-HT: relevance to 5-HT transporters in blood and brain. European journal of pharmacology 674, 337-344, doi:10.1016/j.ejphar.2011.10.033 (2012). 